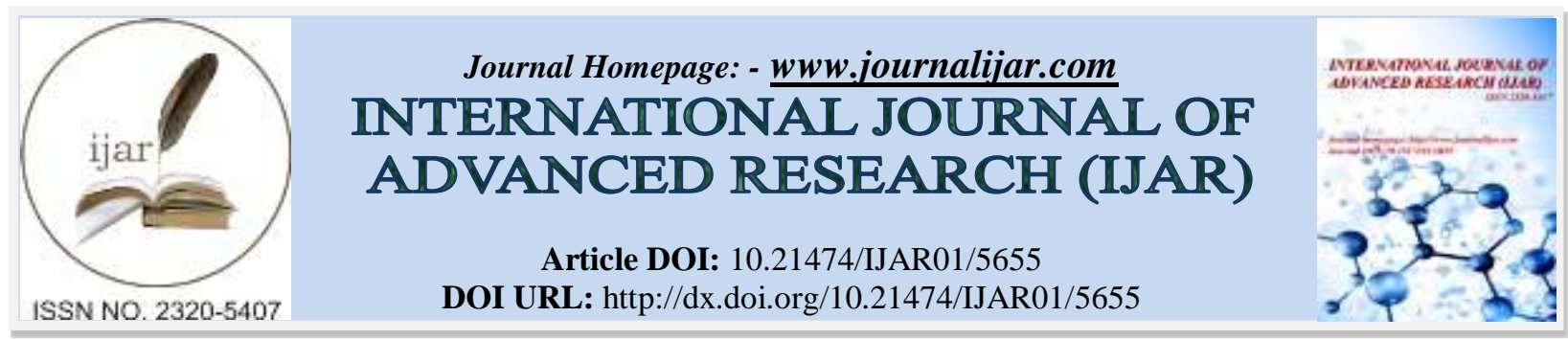

RESEARCH ARTICLE

\title{
ETIO-PATHOPHYSIOLOGICAL PROFILE AND OUTCOME OF CRITICALLY ILL OBSTETRIC PATIENTS ADMITTED TO OBSTETRIC INTENSIVE CARE UNIT AT A TERTIARY CARE OBSTETRIC HOSPITAL IN KASHMIR VALLEY: A RETROSPECTIVE COHORT ANALYSIS.
}

Dr. Syed Masuma Rizvi ${ }^{1}$, Dr. Nadia Nabi ${ }^{2}$, Dr. Mehraj Ud Din ${ }^{3}$ and Dr. Asia Ashraf ${ }^{2}$.

1. Professor Department of Obstetrics and Gynaecology, Lalla Ded Hospital, Government Medical College, Srinagar, India.

2. PG Resident, Department of Obstetrics and Gynaecology, Lalla Ded Hospital, Government Medical College, Srinagar, India.

3. Senior Resident, Department of Anaesthesiology and Critical Care Medicine, Government Medical College, Srinagar, India.

\section{Manuscript Info}

Manuscript History

Received: 18 August 2017

Final Accepted: 20 September 2017

Published: October 2017

Key words:-

Maternal Mortality, High Risk

Pregnancy Obstetric ICU, PIH,

Ecclampsia, Obstetric Hemorrhage, Sepsis.

\section{Abstract}

Objective: To evaluate the reasons for admission and outcomes of obstetric patients admitted to the Intensive Care Unit at the tertiary care obstetric hospital of GMC Srinagar over a period of one year.

Study design: Retrospective cohort analysis.

Setting and Methods: Data of obstetric patients admitted to the Obstetric ICU of Lalla Ded hospital, a tertiary care obstetric hospital of GMC Srinagar, between January 2016 and January 2017 was retrospectively reviewed. Patient demographics, reason for ICU admission, need for ventilatory support and outcome were assessed.

Results: Over the study period, 33,594 patients were admitted to the hospital, of which 31985 were obstetric and 1609 were gynaecology cases. 303 obstetrical patients out of 31985 patients $(0.9 \%)$ needed ICU admission. Out of these ICU admissions, 131 patients (43\%) were referred from other health centers, 101 (33\%) required ventilator support. Nearly half $(43.56 \%)$ were admitted due to severe obstetric hemorrhage, while $37.95 \%$ had pregnancy related hypertension with its complications followed by pregnancy with underlying cardiac disease including Peripartum cardiomyopathy and rheumatic heart diseases and sepsis. Overall mortality within the unit was 19 (6.27\%) during the study period.

Conclusion: Obstetric haemorrhage and complications of pregnancy related hypertension are the leading causes of ICU admission. Early detection with referral to tertiary care centre and involvement of multidisciplinary team with dedicated obstetric intensive care unit is the key to decrease morbidity and improve materno-fetal outcome in high risk pregnancies and in critically ill obstetric patient population.

Copy Right, IJAR, 2017,. All rights reserved. 


\section{Introduction:-}

World Health Organization uses maternal mortality rate as one of the most important indicators of population health and well-being. In India, maternal mortality has declined from 370 deaths per 100,000 live births in 2000 to 190 in 2013 whereas the maternal mortality rate for the United States of America in 2013 was 28 maternal deaths per 100,000 births. ${ }^{[1]}$ Tracking maternal deaths may not be the best way to assess pregnancy-related critical illnesses since the majority of such illnesses do not result in maternal death. As stated by Harmer, "death represents the tip of the morbidity iceberg, the size of which is unknown". [2]

Maternal mortality data collection is well established in many places, but specific surveillance systems that track severe complications of pregnancy not associated with maternal mortality are rare. It has been suggested that most women suffering a critical illness in pregnancy are likely to spend some time in an intensive care unit. ${ }^{[2,3]}$ These cases have been described by some as "near-miss" mortality cases. ${ }^{[4]}$ Therefore, examination of cases admitted to ICU's can provide insight into the nature of pregnancy-related critical illnesses. Determining the etiopathophysiological profile of obstetric patients requiring ICU admissions can help in evaluating the risk factors resulting in obstetric morbidity and mortality while simultaneously help in optimal resource management and allocation especially in low income countries.

Compared to women in their 20s, women aged 35-39 and $\geq 40$ are 2.5 and 5.3 times more likely to suffer from pregnancy-related mortality respectively. ${ }^{[5]}$ WHO regularly systematically reviews maternal mortality worldwide, and in developed countries, 16 percent of maternal deaths were reported to be due to hypertensive disorders of pregnancy. ${ }^{[6]}$ This proportion is greater than the three other leading causes that include hemorrhage (13\%), abortion $(8 \%)$, and sepsis $(2 \%)$. In the USA it is estimated that half of all the maternal deaths are preventable. ${ }^{[7]}$ Between 1998 to 2005, Berg and colleagues reported that 12.3 percent of 4693 pregnancy-related maternal deaths were caused by pre-Ecclampsia or Ecclampsia. ${ }^{[8]}$ The rate was similar to that of 10 percent for maternal deaths reported from France from 2003 through 2007. ${ }^{[9]}$

Despite the reduced maternal mortality rate in India, there is a need to identify the risk factors in obstetric patients that lead to ICU admission so that both maternal mortality and morbidity can be reduced by prevention. Very few studies have been conducted in India evaluating the reason for ICU admissions of critically ill obstetric patients in tertiary hospitals across India. ${ }^{[10-12]}$ The present study was conducted to evaluate the indications for admission, course and outcome of the critically ill obstetric patients in Kashmir valley, in order to evaluate the patient characteristics of critically ill obstetric patients so that high risk obstetric patients are identified early and managed appropriately with early involvement of multidisciplinary obstetric intensive care team.

\section{Methods:-}

The current study was conducted at Lalla Ded Hospital; the tertiary care obstetric hospital of Government Medical College Srinagar, a tertiary care hospital and regional referral center. The study was conducted after approval by institutional ethical committee. Data for this study was collected retrospectively using data from hospital records of the obstetric patients admitted over a period of one year from January 2016 to January 2017 in Lalla Ded hospital, a tertiary care obstetric hospital of Government Medical College Srinagar. This is the only tertiary care level obstetric care hospital in Kashmir Valley. Cases are referred to this hospital from peripheral hospitals and rural areas, and also from local general practitioners all over the valley and adjacent regions.

Data was retrieved from the hospital medical record section and analyzed for determining the age, residence, antenatal registration, Peri-natal period, date of admission and discharge from ICU, pre-admission diagnosis, need for ventilator support and outcome of the patient. All data was anonymised at point of entry into the database. Data retrieved was analyzed using percent fractions and analyzed using STATA 10 (STATACOPR, Texas, USA).

\section{Results:-}

The study was a retrospective observational study from January 2016 to January 2017 conducted at tertiary care obstetric hospital of Government Medical College Srinagar. A total of 33,594 patients were admitted to the hospital during this period. Out of this 31985 admissions were obstetrical patients and 1609 were gynecological cases. 
Table 1 shows the demographic characteristics of the patients admitted to ICU. As shown in the table mean age of the patients was 30.8 years. Eighty-seven percent of the patients belonged to rural areas and 94 percent patients were antenatally booked cases from periphery.

\begin{tabular}{|l|l|l|}
\hline Characteristics & Number of patients & Percentage \\
\hline Age range & 9 & $2.97 \%$ \\
\hline $20-24$ & 110 & $36.30 \%$ \\
\hline $25-29$ & 174 & $57.42 \%$ \\
\hline $30-34$ & 10 & $3.30 \%$ \\
\hline$>35$ & 303 & $100 \%$ \\
\hline Total & \multicolumn{2}{|l|}{} \\
\hline Residence & 39 & $12.87 \%$ \\
\hline Urban & 264 & $87.12 \%$ \\
\hline Rural & \multicolumn{2}{|l|}{} \\
\hline Antenatal Check up & 284 & $93.72 \%$ \\
\hline Booked & 19 & $6.27 \%$ \\
\hline Un-booked & \multicolumn{2}{|l|}{} \\
\hline
\end{tabular}

Table 1 showing demographic characteristics of the patients admitted to ICU.

Indications for the ICU admissions are shown in Table 2. As can be seen from the table 2 the most common cause of admission was obstetric hemorrhage (43.56\%) followed by hypertensive disorders of pregnancy $(37.95 \%)$.

Primary post-partum hemorrhage (21\%) was the most common indication among obstetric hemorrhages followed by abnormal placentation (18\%) with placenta previa and accreta being the commonest causes.

\begin{tabular}{|l|l|l|}
\hline Causes of admission & Number of patients & Percentage \\
\hline Hemorrhages & & $\mathbf{4 3 . 5 6}$ \\
\hline Primary PPH & 59 & 19.47 \\
\hline APH & 49 & 16.17 \\
\hline Uterine Rupture & 13 & 4.29 \\
\hline Ruptured Ectopic Pregnancy & 11 & 3.63 \\
\hline Hypertensive Disorders of pregnancy & & $\mathbf{3 7 . 9 5}$ \\
\hline Gestational Hypertension & 29 & 9.57 \\
\hline Severe Preeclampsia & 32 & 10.56 \\
\hline Ecclampsia & 51 & 16.83 \\
\hline HELLP Syndrome & 1 & 0.33 \\
\hline Cerebro Vascular Accidents & 2 & 0.66 \\
\hline Post-delivery complications & & $\mathbf{1 0 . 2 3}$ \\
\hline Re-exploration post LSCS & 13 & 4.29 \\
\hline Vaginal wall hematoma & 5 & 1.65 \\
\hline Cardiac diseases & 13 & 4.29 \\
\hline Sepsis Related and Others & & $\mathbf{8 . 2 5}$ \\
\hline Sepsis & 8 & 2.64 \\
\hline Septic Abortion & 2 & 0.66 \\
\hline Chorioamnitis & 1 & 0.33 \\
\hline UTI/RTI & 1 & 0.33 \\
\hline Gullian Barrie Syndrome & 2 & 0.66 \\
\hline OTHERS & 11 & 3.63 \\
\hline Total & $\mathbf{3 0 3}$ & $\mathbf{1 0 0}$ \\
\hline Table & & \\
\hline
\end{tabular}

Table 2 showing the disease specific distribution of obstetric patients admitted to the ICU.

During the study period there were 24,489 deliveries representing $73 \%$ of the total admissions out of which 9401 were vaginal deliveries and 15,088 involved deliveries through caesarian section. Out of these 303 patients needed ICU admission representing $1.2 \%$ of the total admissions during the study period. 
During the ICU stay $101(33.33 \%)$ of the patients were intubated and required ventilatory support while rest of the patients were managed without need for mechanical ventilation.

\begin{tabular}{|l|l|l|}
\hline Need for ventilator support & Number of patients & Percentage \\
\hline Yes & 101 & 33.33 \\
\hline No & 202 & 66.66 \\
\hline Total & 303 & 100 \\
\hline
\end{tabular}

Table 3 showing percentage of patients requiring ventilatory support in ICU

$131(43 \%)$ of the patients requiring ICU admission were those who referred from other health centers. Overall $93.72 \%$ of the patients requiring ICU admission survived and were shifted to the recovery ward. A total of 19 $(6.27 \%)$ patients expired in ICU out of which 11 patients were the referred cases.

\begin{tabular}{|l|l|l|}
\hline Outcome & Number of patients & Percentage \\
\hline Survived & 284 & 93.72 \\
\hline Expired & 19 & 6.27 \\
\hline Total & 303 & 100 \\
\hline
\end{tabular}

Table 4 showing outcome of the patients admitted to the ICU.

\section{Discussion:-}

The study was a retrospective observational study from January 2016 to January 2017 conducted at tertiary care obstetric hospital of GMC Srinagar; this is the only tertiary care level obstetric hospital in Kashmir Valley. Cases are referred to this hospital from peripheral district hospitals, and also from local general practitioners all over the valley and adjacent regions.

In our study the mean age of patients admitted in the obstetric intensive care unit was 30.8 years. This was comparable with the study done by Leung et al with mean age $31 \pm 6$ years, Selo-Ojeme et al 29.6 \pm 5.8 years, Baloch et al 28.5 \pm 6.1 years and Saif KM et al 27.3 years. ${ }^{[13-16]}$ However the mean age was higher than the results found in a study conducted by Saha $\mathrm{R}$ et al, which reported $24.5 \pm 4.8$ years. ${ }^{[17]}$

In our study number of booked cases (94\%) was comparable to the study conducted by Gupta S et al showing $79.16 \%$ booked cases. ${ }^{[18]}$ Only $6 \%$ of the cases were unbooked. Whereas the study conducted by Baloch et al had $84.2 \%$ unbooked cases, and Saif KM et al showed $76.3 \%$ unbooked cases. ${ }^{[15}{ }^{16]}$ This finding shows that the number of regular antenatal visits are an important factor in better outcomes rather than the number of booked versus unbooked cases.

The increased number of cases being booked can be attributed to the various Government of India sponsored programs for promoting safe motherhood throughout the country. The objective of this Janani Shishu Suraksha Karyakaram (JSSK) is to reduce maternal and infant mortality by promoting institutional delivery among pregnant women and by providing conditional cash assistance. However, the first contact of these pregnant women is the community health worker (ASHA worker) who may not have the tools to differentiate between high-risk and normal pregnancies. Identifying risk factors that lead to increased SICU admissions could lead to development of protocols that these community workers could use in identifying high-risk cases.

In this study, obstetric hemorrhages (43.56\%) followed by hypertensive disorders of pregnancy (37.95\%) were the two most common causes of obstetrical admission to the SICU. These two causes were also the common causes of ICU admission in studies conducted by Selo - Ojeme et al (36.4\% and 39.4\%) Baloch et al (38.2\% and 36.2\%), Bhat $P$ et al $(27.7 \%$ and $26.2 \%)$, and Saif KM et al $(22.8 \%$ and $17.4 \%) .{ }^{[14-16,19]}$

Other reasons for admission to the obstetric ICU included pregnancy with cardiac diseases (4.29\%) including Peripartum cardiomyopathy and rheumatic heart diseases followed by sepsis and patients with septic abortions (3.3\%). Overall the mortality was $7.26 \%$ which correlates well with other studies which have shown mortality figures to range from $2 \%$ to $20 \%$ worldwide. ${ }^{[1213,18]}$ 
Majority of these critically ill obstetric patients are young with good physiological reserves and the mortality and morbidity is avoidable if identified early and managed aggressively in dedicated obstetric intensive care units and high dependency units. Optimal management of such patients requires early recognition of high risk women based on their clinical risk factors, with accurate preoperative diagnosis, good maternal counselling and planning of delivery with involvement of experienced obstetricians, anaesthesiologists and intensivist and other multidisciplinary team members including neonatologists, haematologists, interventional radiologists nurses and midwifes.

Being the only tertiary care hospital in Kashmir, our hospital caters to a large population and the patients being referred can take over 2 hours to reach the hospital, increasing maternal and fetal morbidity and mortality.

In our low resource setting, the failure to recognize the need for antenatal care, means many patients with serious obstetric disorders are either not antenatally booked, or booked with health workers who cannot recognize high risk pregnancies, or diagnosis is delayed till they suffer serious irreversible organ damage.

Referral management systems needs to be improvised in order to improve and streamline communication amongst primary care physicians, specialists and any other health care providers in order to achieve better patient care. Structured, practical, pragmatic and community based protocols should be developed to train community workers, obstetric health care workers and doctors to identify and manage risk factors at an early stage to reduce obstetric ICU admissions and improve patient outcomes.

Further studies are however needed and should be encouraged to accurately describe and categorize the disease severity, course and outcome of these group of patients so that protocols are developed for early identification of high risk factors and appropriate management strategy is adopted in a timely fashion to decrease maternal morbidity and mortality.

\section{Conclusion:-}

In our study, the most common indications for ICU admissions in the obstetric population were obstetric haemorrhage and complications of pregnancy related hypertension. Morbidity and mortality associated with these could be reduced by prevention, early detection and involvement of multidisciplinary team with increased access to dedicated obstetric intensive care unit and high dependency units. Timely identification and referral to tertiary care centre is the key to decrease morbidity and improve materno-fetal outcome in high risk pregnancies and in critically ill obstetric patient population.

Funding:- None

Conflict of interest:- None declared

Ethical approval:- Approval by institutional ethical committee

\section{References:-}

1. Trends in maternal mortality: 1990 to 2013. Estimates by WHO, UNICEF, UNFPA, The World Bank and the United Nations Population Division. Geneva: World Health Organization; 2014. Available at: http://www.who.int/reproductivehealth/publications/monitoring/maternal-mortality-2013/en/ . Accessed 1 August 2017.

2. Harmer M Maternal mortality - is it still relevant? Anaesthesia $1997 ; 52: 99-100$.

3. MahutteNG, Murphy - Kaulbeck L, Le Q, Solomon J , Benjamin A, Boyd ME . Obstetrics admissions to the intensive care unit .ObstetGynecol $1999 ; 94: 263$ - 266.

4. Tunçalp O, Hindin MJ, Souza JP, Chou D, Say L. The prevalence of maternal near miss: a systematic review. BJOG 2012 May;119(6):65-61.

5. Geller SE, Cox SM, Callaghan WM, Berg CJ: Morbidity and mortality in pregnancy: laying the groundwork for safe motherhood. Womens Health Issues 2006 16:176

6. Khan KS, Wojdyla D, Say L, Gülmezoglu AM, Van Look Paul F A: WHO analysis of causes of maternal death: a systematic review. Lancet 2006: 367:1066

7. Main EK, Menard MK. Maternal mortality: time for national action. Obstet Gynecol. 2013 Oct;122(4):735-6

8. Berg CJ, Callaghan WM, SwersonC,Henderson Z: Pregnancy-related mortality in the United States, 1998 to 2006. ObstetGynecol2010: 116(6):1302 
9. Saucedo M, Deneux-Tharaux C, Bouvier-Colle MH: Ten years of confidential inquiries into maternal deaths in France, 1998-2007. ObstetGynecol2013, 122(4):752.

10. Ashakiran T. Rathod and Malini K.V, "Study of Obstetric Admissions to the Intensive Care Unit of a Tertiary Care Hospital," Journal of Obstetrics and Gynecology of India, vol. 66, pp. 12-17, 2016.

11. Ashraf N, Mishra SK, Kundra P, SoundaraghavanS, and Habeebullah S-Obstetric Patients Requiring Intensive Care: A One Year Retrospective Study in a Tertiary Care Institute in India, Anesthesiology Research and Practice Volume 2014Article ID 789450

12. Dasgupta S, Jha T, Bagchi P, Singh SS, Gorai R, Choudhury SD. Critically Ill Obstetric Patients in a General Critical Care Unit: A 5 Years' Retrospective Study in a Public Teaching Hospital of Eastern India. Indian Journal of Critical Care Medicine :2017;21(5):294-302.

13. Natalie YW Leung ,Artur CW Lau,Kenny Kc Chan,WW Yan;clinical characteristics and outcomes of obstetric patients admitted to the intensive care unit; a 10 year retrospective review 2010;Hong Kong J;Vol 16,NO 1.Page 18-25.

14. Selo - Ojeme DO, Omosaiye M, Battacherjee P, Kadir RA . Risk factors for obstetric admissions to the intensive care unit in a tertiary hospital: a case control study. Arch Gynecol Obstet $2005 ; 272: 207$.

15. Baloch R, Jakhrani NK, Zeb E, Hafeez S, Abassi M, Abassi FN. Pattern and outcome of obstetric admissions to the surgical intensice care unit- a ten year study. J Surg Pak (International). 2010 Oct-Dec;15(4):171-76.I

16. Saif KM, Tahmina S, Maitree P. A prospective study of clinical profile and outcome of critically ill obstetric patients in ICU at a tertiary level hospital in India. Anaesth Pain \& Intensive Care 2013;17(3):243-247

17. Saha R, Shakya A, Study of obstetric patients admitted to Intensive Care Unit (ICU) at Kathmandu Medical College Teaching Hospital Journal of Kathmandu Medical College, Vol. 2, No. 4, Issue 6, Oct.-Dec., 2013 196200.

18. Gupta S, Naithani U, Doshi V, Bhargavi V, Vijay BS; Obstetric critical care :a prospective analysis of clinical characteristics predictability, and fetomaternal outcome in a new dedicated obstetric intensive care unit; mar apr 2011; Indaian J od Anaesthesia;Vol.55;Issue 2.

19. Poornima B. Ramachandra Bhat, Mahesha H. Navada, Sujaya V. Rao, and G. Nagarathna Evaluation of obstetric admissions to intensive care unit of a tertiary referral center in coastal India Indian J Crit Care Med. 2013 JanFeb;17(1): 34-37 